\title{
ANOTHER THEOREM ON CONVEX COMBINATIONS OF UNIMODULAR FUNCTIONS
}

\author{
BY STEPHEN FISHER
}

Communicated by Wolfgang Wasow, April 9, 1969

Let $R$ be a finite open Riemann surface; that is, $R$ is obtained by deleting from a compact Riemann surface a finite number of disjoint closed discs, each of which has an analytic simple closed curve as boundary. Let $A(R)$ be the algebra of functions which are continuous on the closure of $R$ and analytic on $R ; A(R)$ is a Banach space under the supremum norm. An element $f$ of $A(R)$ will be called inner if $|f|=1$ on the boundary of $R$. The following theorem extends the author's earlier result, where $R$ was the unit disc in the complex plane [3].

TheOREm. The closed convex hull of the inner functions in $A(R)$ is the unit ball of $A(R)$.

The proof requires two lemmas whose proofs will be given after the proof of the theorem.

Lemma 1. Let $z_{1}, \cdots, z_{N}$ be distinct points of $R$ and let $h$ be an analytic function on $R$ bounded by 1 . Then there is an inner function $f$ in $A(R)$ with $f\left(z_{j}\right)=h\left(z_{j}\right)$ for $j=1, \cdots, N$.

Lemma 2. Let $E$ be a compact subset of the boundary of $R$ of zero harmonic measure and let $\mu$ be a positive regular Borel measure on $E$. If $g$ is a continuous function on $E$ of unit modulus, then there is a sequence $\left\{f_{n}\right\}$ of inner functions in $A(R)$ such that

(i) $f_{n}$ converges to $g$ a.e. $\mu$ and

(ii) $f_{n}$ converges uniformly to one on compact subsets of $R$.

Proof of The THeorem. Let $Q$ be the closed convex hull of the inner functions in $A(R)$. By the basic separation theorem [2, V.2.10] if $Q$ were not equal to the unit ball of $A(R)$, there would be a measure $\lambda$ which strictly separated $Q$ from some element of the unit ball of $A(R)$. By [1, Corollary 5] the set of linear functionals on $A(R)$ which attain their norm at some element of the unit ball of $A(R)$ is dense in the dual space of $A(R)$. Hence, it suffices to prove this: if $\lambda$ is a measure on $B$, the boundary of $R$, with $\|\lambda\|=1=\int f d \lambda$, some $f \in A(R)$, $\|f\|=1$, then $\sup \left\{\operatorname{Re} \int q d \lambda: q \in Q\right\}=1$.

Such a measure $\lambda$ has the form

$$
d \lambda=\bar{f} g d m+\bar{f} d \mu
$$


where $m$ is harmonic measure for some fixed point $p$ in $R, \mu$ is nonnegative and singular with respect to $m, g$ is nonnegative, and the closed support of $\lambda$ lies in the set where $f$ has unit modulus. By choosing a sufficiently large compact subset of the support of $\mu$ we may also assume that the support of $\mu$ is compact and, of course, has zero $m$-measure.

If $z \in R$ let $P_{z} d m$ be the harmonic measure for $z$ on $B$. It is easy to see that the linear span of the set $\left\{P_{z}: z \in R\right\}$ is dense in $L^{1}(B, m)$. Hence, given $\epsilon>0$, there are points $z_{1}, \cdots, z_{N}$ in $R$ and constants $c_{1}, \cdots, c_{N}$ with $\left\|\sum_{i=1}^{N} c_{i} P_{i}-g \bar{f}\right\|<\epsilon$, where we have written $P_{i}$ for $P_{z_{i}}$. Thus $0 \leqq \int g d m=\int f f g d m \leqq \operatorname{Re}\left(\sum_{i=1}^{N} c_{i} f\left(z_{i}\right)\right)+\epsilon$.

By Lemma 1 there is an inner function $I$ in $A(R)$ with $I\left(z_{j}\right)=f\left(z_{j}\right)$ for $j=1, \cdots, N$. By Lemma 2 , there is a sequence $\left\{f_{n}\right\}$ of inner functions in $A(R)$ with $f_{n} \rightarrow \bar{I} f$ a.e. $\mu$ and $f_{n}\left(z_{j}\right) \rightarrow 1$ for $j=1, \cdots, N$. Let $h_{n}=I f_{n}$; then $h_{n}$ is an inner function in $A(R)$ for each $n, h_{n}\left(z_{j}\right)$ $\rightarrow f\left(z_{j}\right)$ for $j=1, \cdots, N$ and $h_{n} \rightarrow f$ a.e. $\mu$. Hence,

$$
\begin{aligned}
\operatorname{Re} \int h_{n} d \lambda & =\operatorname{Re}\left(\int h_{n} \bar{f} g d m\right)+\operatorname{Re}\left(\int h_{n} \bar{f} d \mu\right) \\
& \geqq \operatorname{Re}\left(\int h_{n}\left(\sum_{1}^{N} c_{i} P_{i}\right) d m\right)-\epsilon+\operatorname{Re}\left(\int h_{n} \bar{f} d \mu\right) \\
& =\operatorname{Re}\left(\sum_{1}^{N} c_{i} h_{n}\left(z_{i}\right)\right)-\epsilon+\operatorname{Re}\left(\int h_{n} \bar{f} d \mu\right) \\
& \geqq \int g d m-3 \epsilon+\int d \mu-\epsilon=1-4 \epsilon
\end{aligned}
$$

for $n$ sufficiently large. This establishes the theorem.

Proof of Lemma 1. This a result of Heins [4, p. 571].

Proof of Lemma 2. By a theorem of Stout [7, Theorem IV. 1] there are three inner functions in $A(R)$, say $h_{1}, h_{2}$, and $h_{3}$, which separate the points of the closure of $R$ and whose differentials have no common zero on $R . h_{i}(E)$ is a compact subset of the unit circle of arc length zero for $i=1,2,3$. Let $H$ embed $R$ in the unit three-polydisc by $H(z)=\left(h_{1}(z), h_{2}(z), h_{3}(z)\right)$, and let $F=H(E)$. Since $F$ is a compact subset of $h_{1}(E) \times h_{2}(E) \times h_{3}(E)$, it is a peak-interpolation set for the polydisc algebra [6, Theorem 4.1]. Choose a function $G$ in the polydisc algebra which is bounded by one and satisfies $G(H(z))=g(z)$ for $z \in E$. Given $\epsilon>0$ there are by Rudin's theorem [5, see final Remark] unimodular functions $U_{1}, \cdots, U_{k}$ in the polydisc algebra and positive numbers $\lambda_{1}, \cdots, \lambda_{k}$ which sum to 1 such that $\left\|\sum_{1}^{k} \lambda_{j} U_{j}-G\right\|<\epsilon$. 
Let $V_{j}=U_{j} \circ H$. Then $V_{j}$ is an inner function in $A(R)$ and $\left\|\sum_{1}^{k} \lambda_{j} V_{j}-g\right\|_{E}<\epsilon$. This implies that there are inner functions $f_{n}$ in $A(R)$ such that $\int f_{n} \bar{g} d \mu \rightarrow 1$. (We are assuming that $\|\mu\|=1$; this involves no loss of generality.) Hence

$$
\begin{aligned}
\int\left|f_{n}-g\right|^{2} d \mu & =\int\left|f_{n}\right|^{2}+\int|g|^{2} d \mu-2 \operatorname{Re} \int f_{n} \bar{g} d \mu \\
& =2-2 \operatorname{Re} \int f_{n} \bar{g} d \mu \rightarrow 0 .
\end{aligned}
$$

Let $z_{0} \in R$; by Lemma 1 there is an inner function $I$ in $A(R)$ with $I\left(z_{0}\right)=0$. By the above we can find inner functions $f_{n}$ in $A(R)$ with $f_{n}$ converging in $L^{2}(\mu)$ to $\bar{I} g$. Thus $I f_{n}$ is inner and in $A(R)$ for each $n$ and $I f_{n}$ converges to $g$ in $L^{2}(\mu)$ and vanishes at $z_{0}$. Finally, choose a sequence of numbers $\left\{\beta_{n}\right\}$ with $0<\beta_{n}<1$ and $\beta_{n} \rightarrow 1$. For each $n$ there is an inner function $g_{n}$ in $A(R)$ with

(i) $g_{n}\left(z_{0}\right)=0$ and

(ii) the $L^{2}(\mu)$ distance from $g_{n}$ to $\left(g-\beta_{n}\right)\left(1-\beta_{n} g\right)^{-1}$ is less than $\left(1-\beta_{n}\right)^{2}$.

If we put $f_{n}=\left(g_{n}+\beta_{n}\right)\left(1+\beta_{n} g_{n}\right)^{-1}$, then $f_{n}$ is in $A(R)$, is inner, has the value $\beta_{n}$ at $z_{0}$, and finally, the $L^{2}(\mu)$ distance from $g$ to $f_{n}$ is no more than $2\left(1-\beta_{n}\right)$. Since the $f_{n}$ are bounded by 1 and converge to 1 at an interior point of $R$, they must converge uniformly to 1 on compact subsets of $R$. A subsequence converges a.e. $\mu$ to $g$.

\section{REFERENCES}

1. E. Bishop and R. R. Phelps, The support functionals of a convex set, Proc. Sympos. Pure Math., vol. VII, Amer. Math. Soc., Providence, R.I., 1963, pp. 27-35.

2. N. Dunford and J. T. Schwartz, Linear operators. Part I, Interscience, New York, 1957.

3. S. Fisher, The convex hull of the finite Blaschke products, Bull. Amer. Math. Soc. 74 (1968), 1128-1129.

4. M. Heins, A lemma on positive harmonic functions, Ann. of Math. (2) 52 (1950), 568-573.

5. W. Rudin, Convex combinations of unimodular functions, Bull. Amer. Math. Soc. 75 (1969), 795-797.

6. W. Rudin and E. L. Stout, Boundary properties of functions of several complex variables, J. Math. Mech. 14 (1965), 991-1006.

7. E. L. Stout, On some algebras of analytic functions on finite open Riemann surfaces, Math. Z. 92 (1966), 366-379.

NorthWESTERN UnIVERSITy, Evans'TON, IlLINOIS 60201 